\title{
Robot Vacuum Cleaner Personality and Behavior
}

\author{
Bram Hendriks • Bernt Meerbeek · Stella Boess • \\ Steffen Pauws • Marieke Sonneveld
}

Accepted: 14 November 2010 / Published online: 23 December 2010

(c) The Author(s) 2010. This article is published with open access at Springerlink.com

\begin{abstract}
In this paper we report our study on the user experience of robot vacuum cleaner behavior. How do people want to experience this new type of cleaning appliance? Interviews were conducted to elicit a desired robot vacuum cleaner personality. With this knowledge in mind, behavior was designed for a future robot vacuum cleaner. A video prototype was used to evaluate how people experienced the behavior of this robot vacuum cleaner. The results indicate that people recognized the intended personality in the robot behavior. We recommend using a personality model as a tool for developing robot behavior.
\end{abstract}

Keywords Robot vacuum cleaner · User experience · Personality $\cdot$ Behavior

B. Hendriks $(\varangle) \cdot$ S. Boess $\cdot$ M. Sonneveld

Delft University of Technology, Delft, The Netherlands

e-mail: mail@bramhendriks.nl

S. Boess

e-mail: s.u.boess@tudelft.nl

M. Sonneveld

e-mail: m.h.sonneveld@tudelft.nl

B. Hendriks $\cdot$ B. Meerbeek $\cdot$ S. Pauws

Philips Research, Eindhoven, The Netherlands

B. Meerbeek

e-mail: bernt.meerbeek@philips.com

S. Pauws

e-mail: steffen.pauws@philips.com

\section{Introduction}

\subsection{A New Kind of Presence}

Robot vacuum cleaners have been on the market for a few years now and, to an increasing extent, these products are taking over our chores. Robot vacuum cleaners are vacuum cleaners that clean floorings autonomously, and are among the first service robots that enter our homes. This type of vacuum cleaner is a new kind of presence to get used to. It exhibits autonomous behavior and it moves around our homes and affects our daily lives. This is very different from conventional, non-robotic vacuum cleaners. People are no longer in full control of what the product is doing, where and when. People need to understand and trust the robot vacuum cleaner. Crucial for the user experience and acceptance of the product is the design of appropriate interaction between human and robot [1].

Although robot vacuum cleaners are a relatively new phenomenon, the idea in itself-having a robot cleaning assistant at home-is not. People have dreamt about it for decades. Back in the seventies Quasar Industries claimed to have developed a robot capable of carrying out chores in the home [2]. It turned out to be a fake. But today, more than thirty years later, a variety of robot vacuum cleaners is on the market. These are developed by Electrolux, iRobot, Hanool, LG and Samung, among others [3]. Since robots are entering our homes now, it is interesting to study how users experience this new kind of presence in their domestic environment.

\subsection{Personality}

People tend to behave towards artifacts in a social way, particularly if artifacts exhibit some degree of autonomy such 
Fig. 1 From desired personality to evaluation of personality
Context

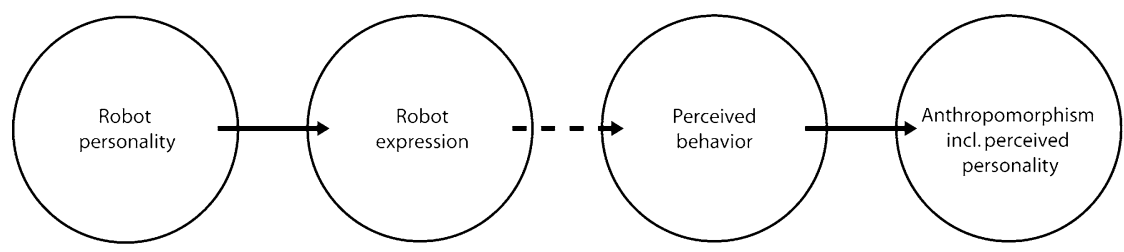

Robot vacuum cleaner

User experience as robot vacuum cleaners [4-9]. Aspects of anthropomorphism - the attribution of human qualities to non-humansare reported in studies on the experience of robot vacuum cleaners. The most studied robot vacuum cleaner is the iRobot Roomba. It is reported that people name it, and that people ascribe a gender and personality to Roomba [4-9]. The personality of Roomba is sometimes described as stubborn, silly [5], crazy [6], intelligent [8] and dumb [9].

An accepted and widely used model that describes personality is the Five-Factor Model (FFM) of personality, also referred to as the Big Five. This model organizes personality traits in terms of five basic dimensions: neuroticism, extraversion, openness to experience, agreeableness and conscientiousness [10]. A number of instruments-or inventories - capable of measuring personality exist, among which: NEO PI-R [11] and TIPI [12]. Although these are inventories used to study personality in a systematic way, people assess personality on a daily basis and form an impression of others, in order to know what to expect [13]. But in their daily lives, people do not only use human personality characteristics to describe people, they also use these to describe products [13] and interaction with products [14]. Or more specific: interaction with computers [15], robots [16] and-as we have seen - even robot vacuum cleaners [4-7]. Here, we refer to personality as the use of human personality characteristics to describe a robot vacuum cleaner.

Studies have shown that people prefer more extraverted and agreeable personalities over more introverted and formal ones when interacting with a computer [15] and a robot [16]. But these studies by Reeves and Nass [15], and Meerbeek, Hoonhout, Bingley and Terken [16] focus on applications with a social function, whereas a robotic vacuum cleaner primarily serves service purposes.

\subsection{Study Design and Research Questions}

This study consists of two parts. First, we investigate what kind of robot vacuum cleaner personality people desire. How do people want the personality of their robot vacuum cleaner to be? This personality is then used to develop a video prototype-a mock-up in the form of film - of robot vacuum cleaner behavior. The personality serves as a design guideline, as suggested by Meerbeek, Saerbeck and
Bartneck [1], and is a high-level description of the way in which the robot interacts with its environment. A welldefined and clearly communicated personality can serve as a mental model of the robot for users and so facilitate the interaction [17].

Second, we investigate how people experience the personality of the robot vacuum cleaner as presented in the video prototype. Does the personality of the robot vacuum cleaner they experience match with the desired personality? The relationship between the two parts of the study is shown in Fig. 1. A similar approach is suggested by Young, Hawkins, Sharlin and Igarashi: designers could use robot expressions as a means to influence perception, in an attempt to convey certain robot characteristics [7].

\section{Desired Robot Vacuum Cleaner Personality}

\subsection{Research Method}

In order to find out what robot vacuum cleaner personality people desire, a semi-structured interview was done with six participants, two women and four men. They were selected because they were likely to be early adopters of robot vacuum cleaners. The participants share some distinctive characteristics. They are all busy scheduled, and either have a background in technology or have affinity with technology. Besides this, they are Dutch. Before the actual interview, all participants were familiarized with the concept of a robot vacuum cleaner by explaining the product and presenting a visual overview of various robot vacuum cleaners. For the interview, thirty personality characteristics were adopted from existing Big Five personality inventories $[11,12]$. For each of the five dimensions of the Big Five, six characteristics that were considered relevant and interesting with respect to robot vacuuming cleaning were selected. Of these six characteristics, three have a positive connotation towards the dimension, whereas the other three have a negative connotation. The characteristics were randomly presented on cards, and the participants were asked to evaluate the desirability. The personality inventories were used as a starting point, and the selected characteristics were cues to talk about the desired robot personality and behavior. It was 
Fig. 2 Extent to which the personality characteristics are desired
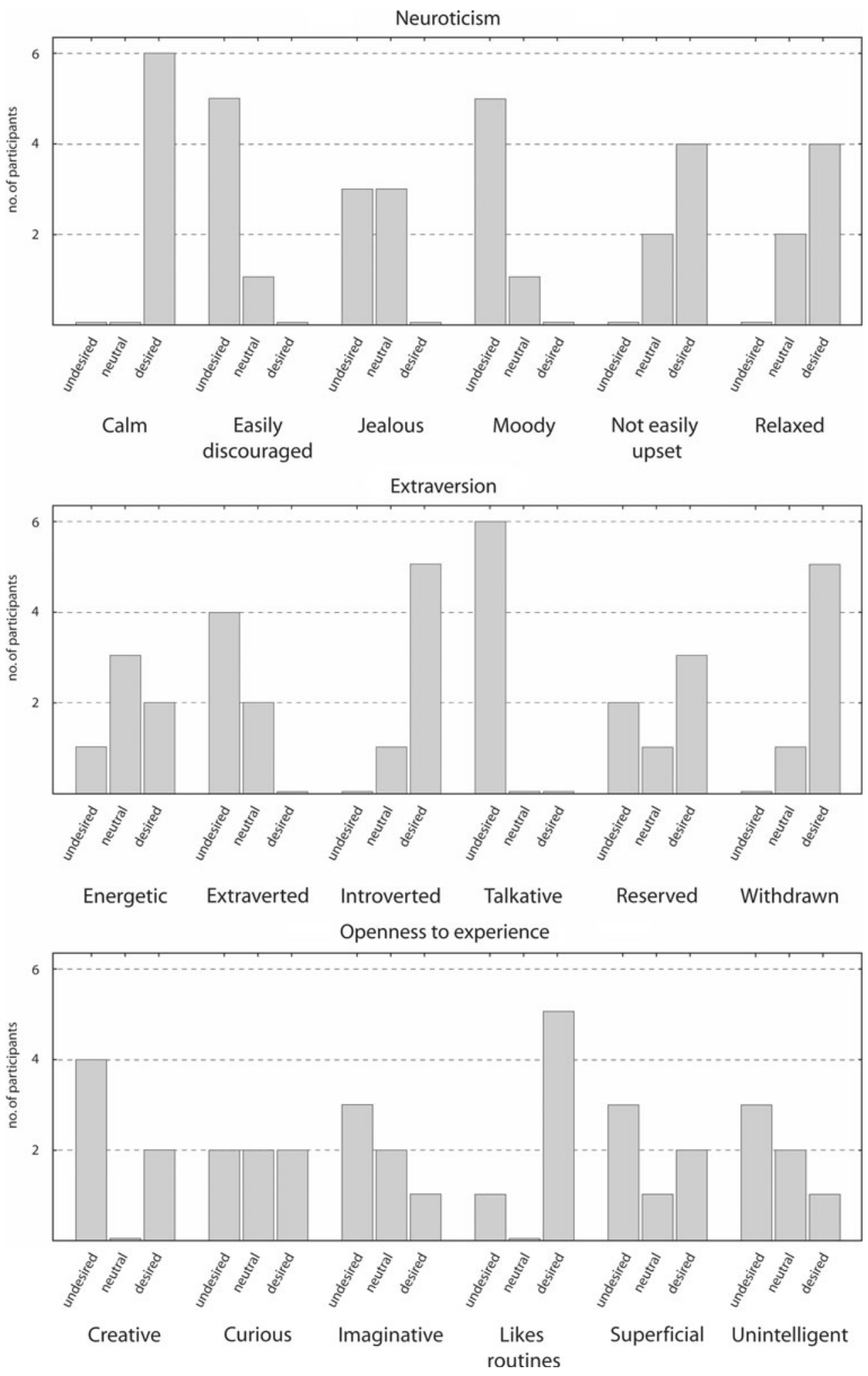

found that people could quickly and easily assess the presented characteristics, and that they could make imaginative descriptions of their desired robot vacuum cleaner's personality. An overview of the selected characteristics is presented in Fig. 2.

\subsection{Results}

We discuss the most relevant results with respect to the desired personality here. The participants desired a calm robot vacuum cleaner (6 participants out of 6); it should ex- 
Fig. 2 (Continued)
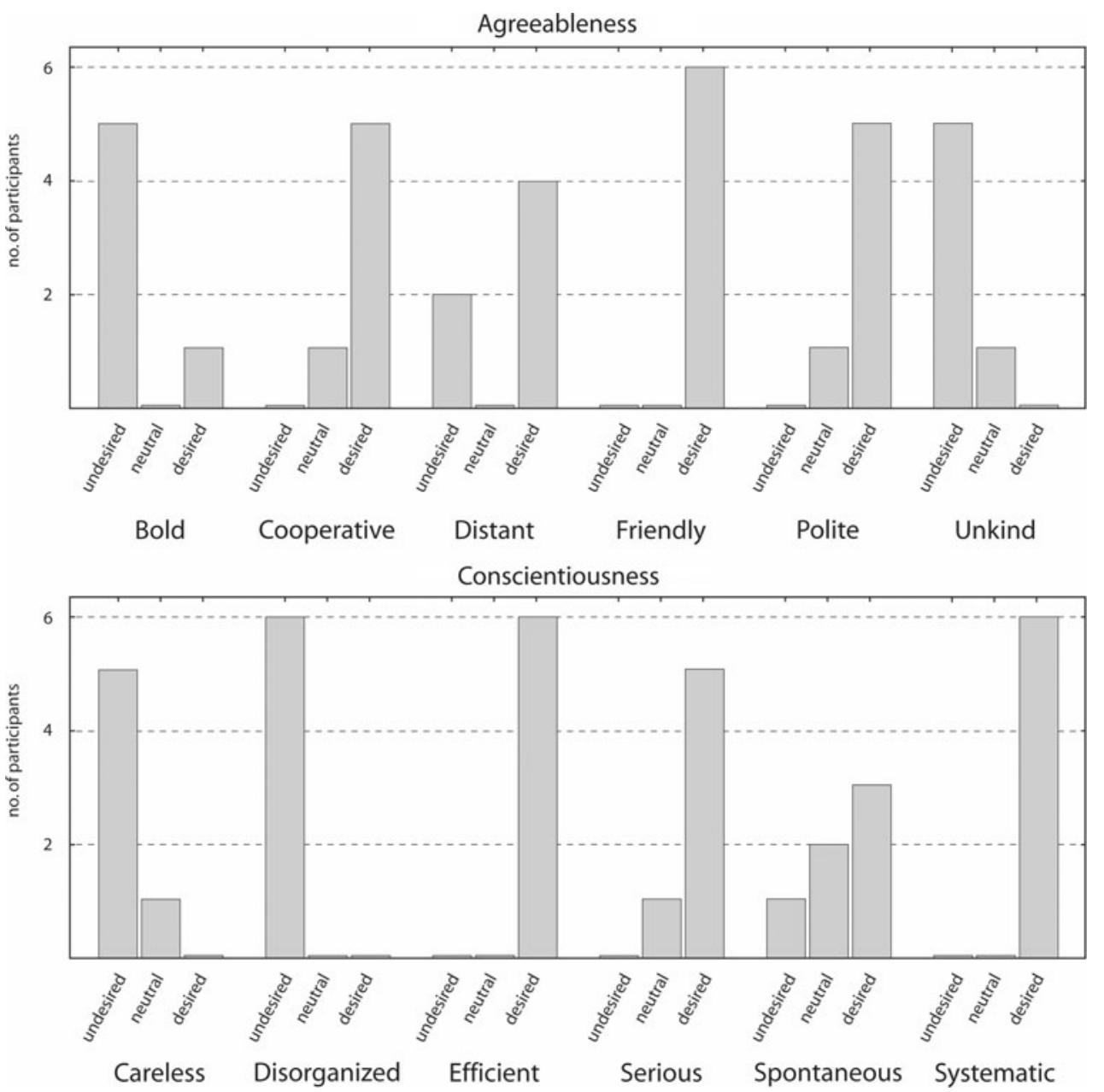

press that it is in control of the situation. Furthermore, they wanted the robot vacuum cleaner to be cooperative in nature (5 participants). All of them desired an efficient robot vacuum cleaner (6 participants). They wanted it to like routines (5 participants), as vacuuming is very much a routine job. The participants desired a polite robot vacuum cleaner (5 participants), as well as one that behaves in a systematic way (6). To summarize the above, the interviews made clear that participants did not desire fancy gadgetry, but a robot vacuum cleaner that fulfills its cleaning task. Figure 2 provides an overview of the results of the interview. The interviews resulted in more than just quantitative data. It resulted in rich information on what a certain personality characteristic could mean for the robot behavior, according to the participants. For example, a calm robot vacuum cleaner was considered not to make a lot of noise and not to disturb unnecessarily.

In addition to the characteristics as presented in Fig. 2, people also brought up additional desired and undesired robot vacuum cleaner personality characteristics. Examples of these additional characteristics are grouped by dimension and listed in Table 1.

\section{Design of the Video Prototype}

The results from the interviews were used as a basis for the robot vacuum cleaner behavior. This behavior was presented by means of a video prototype, which was recorded at the Philips Research ExperienceLab. A part of this observational laboratory is arranged as a living room [18], and was therefore used to mimic real human-robot interactions in a domestic setting. Paper-based methods are perfectly suited to explore a first series of ideas for personality and behavior. But these ideas are hard to capture on paper, especially the subtleties that are so important in interaction. Full implementation of a working robot is often too costly and time consuming. Video prototyping on the other hand, is a very suitable way for studying human-robot interaction [19] and could lead to results that are comparable to those that could be obtained from live interactions with the robot [20]. In a study by Walters, Syrdal, Dautenhahn, Boekhorst and Koay [20] the results obtained from participants watching another person interacting with a robot were statistically comparable to those obtained from participants who interacted with 
Table 1 Personality dimensions along with examples of characteristics brought up by participants

\begin{tabular}{llll}
\hline Dimension & Characteristic & Dimension & Characteristic \\
\hline Neuroticism & Not depressed & Agreeableness & Helpful \\
& Timid & & Helpfullness \\
Extraversion & Cool & & Kind \\
& Not cuddlesome & & Not irritating \\
& Not too present & & Not sneaky \\
& Shy & & Self-willed \\
& Silent & & Serving \\
& Solitary & Hard-working \\
& Dumb & & Neat \\
& No-nonsense & & \\
\hline
\end{tabular}

a real robot. With a video prototype it is impossible however, to study long-term interaction experiences [4].

The five-minute video prototype comprises a conceptual robot vacuum cleaner that encounters a variety of situations at home. Situations such as recharging the batteries and vacuuming dirty spots. The many situations a robot vacuum cleaner may encounter at home trigger different behavior, depending on its personality. These possible situations were discussed in focus groups-groups of possible end users of the robot vacuum cleaner-and a set of ten situations was selected for implementation in the video prototype. In each of these situations the behavior of the robot vacuum cleaner is based on the desired personality characteristics.

The translation from personality to behavior was inspired by a role play in which a group of actors was asked to act like a robot vacuum cleaner with these desired characteristics. Role playing was used as a way of accessing the experience of an interaction. Its use contributes to the quality of interaction in a product to be designed [21]. A similar processfrom a desired robot vacuum cleaner personality to behavior through role playing — was followed by Meerbeek, Saerbeck and Bartneck [1]. Attributes, such as macaroni, were available to support acting out some of the situations (e.g. 'cleaning a dirty spot'). An introductory exercise was meant to familiarize the actors with the personality. Then, the actors were asked to act out situations-as if they were the robot vacuum cleaner-making use of motion and sound (expression through light was taken into consideration only after this exercise). In general, the actors either crawled about or walked around at a slow pace to imitate a vacuum cleaner. Often, a typical vacuuming sound was simulated by them.

In this study, the robot vacuum cleaner behavior focuses on expression through motion, sound and light. These ways of expression seem most relevant for robot vacuum cleaner behavior. The different modalities all add up to the experience of the behavior, and strengthen each other. See Table 2, for an overview of the modalities and dimensions of these modalities, along with some examples of possible effects.
Table 2 Modalities and dimensions of expression, including examples

\begin{tabular}{lll}
\hline Modality & Dimension & Examples of effects \\
\hline Motion & Spatiality & $\begin{array}{l}\text { Directions, paths and planes, big-small } \\
\text { Fast-slow, accelerating-decelerating, } \\
\end{array}$ \\
& Time & $\begin{array}{l}\text { regular-irregular } \\
\text { Tension-release, control-uncontrolled }\end{array}$ \\
& Force & Intensity, high-low \\
Sound & Loudness & Frequency, high-low \\
& Pitch & Tonal-noise \\
& Timbre & On-off, fast-slow, regular-irregular \\
\hline
\end{tabular}

The dimensions are based on studies by Klooster and Overbeeke [22], and Van Egmond [23]. Motion is defined by its dimensions of spatiality, time and force. Sound by loudness, pitch and timbre. Light is defined by time. Table 2 was used as a way to describe behavior in a systematic way. The role play - which was captured on film-was analyzed, and for every situation the behavior was defined and described by means of the modalities and dimensions as listed in the table.

The behavior of the actors was adopted in an abstract way, as, of course, human behavior cannot be translated to a robot vacuum cleaner directly. For example, one of the actors who was asked to act like a robot vacuuming a dirty spot, started to clean that spot slowly thereby making repetitive, firm movements back and forth. These motion aspects of the behavior were then described as taking place on a small plane, slow, regular and tensed.

These descriptions were then used for the development of variations of robot vacuum cleaner behavior. Videos of variations of robot vacuum cleaner behavior were evaluated by six possible end users of the product. The participants were asked, while keeping the desired personality in mind, which variations of behavior they preferred over another 
Fig. 3 Impression of the video prototype
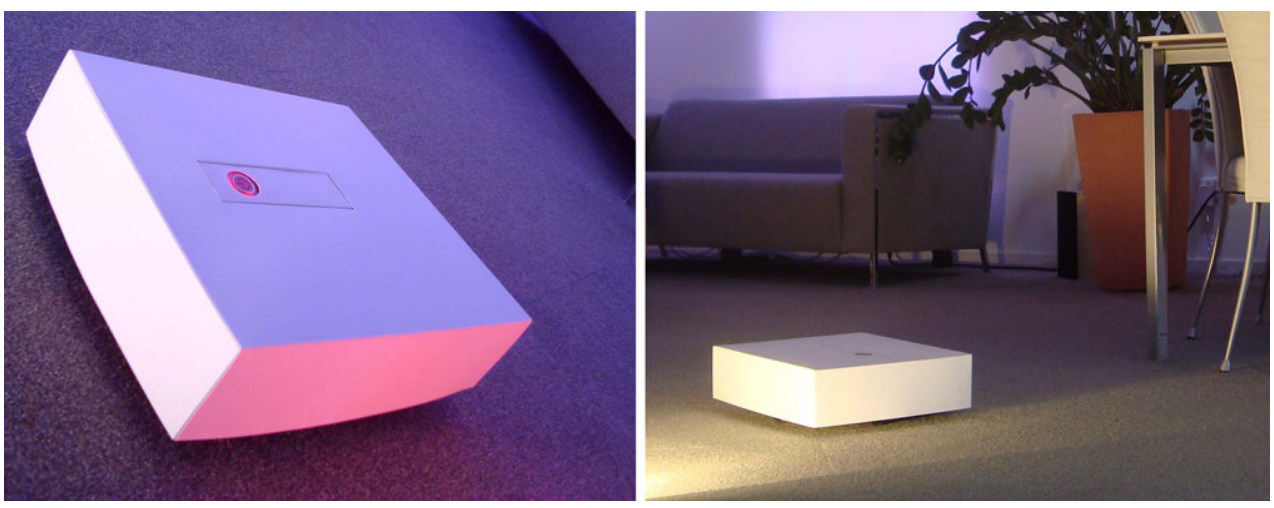

and to indicate why they did so. Improvements for the designed behaviors were brought up by the participants too. The preferred and improved behaviors were combined in a final video prototype of robot vacuum cleaner behavior on the basis of the desired robot personality.

The robot vacuum cleaner in the video is represented by simply a cardboard box, as in this study the focus is on behavior. Elements, such as an on-off button, were added to the appearance of the robot vacuum cleaner, as these were thought to be essential for the behavior as shown in the video prototype. An iRobot Roomba robot vacuum cleaner was used as a test platform for the motion aspects of the behavior to be designed. This Roomba was manually controlled by means of a joystick via a Bluetooth connection. A microcontroller was used to control the light, which consists of six separate LEDs. The vacuuming sound of the robot vacuum cleaner was recorded from a conventional vacuum cleaner, whereas additional sounds were designed by using sound development software. See Fig. 3 for an impression of the video prototype.

\section{Experienced Robot Vacuum Cleaner Personality}

\subsection{Research Method}

While evaluating the video prototype, we looked for signs of anthropomorphism and the perception of personality in particular. We therefore asked fifteen participants-eight women and seven men who fit into the same target group as described above-to think out loud while they watched the video prototype, and to describe their overall impression of the robot vacuum cleaner behavior afterwards. To investigate whether the perceived personality matched with the intended personality, we asked the participants to indicate what kind of personality the robot in the video prototype exhibits. For each of the thirty personality characteristics (the same as those used during the interviews), participants indicated on a rating scale, ranging from 1 (strongly disagree) to 5 (strongly agree), to what extent they thought these applied to the robot as seen in the video. All participants completed this task individually, after having seen the complete video prototype.

\subsection{Results}

When the fifteen participants were asked for their overall impression of the robot vacuum cleaner behavior, they described it by using personality characteristics such as appropriate (3 participants out of 15), calm (3), boring (2), careful (2), and systematic (2). It was probably experienced as boring, as the robot simply vacuumed the living room in a systematic way. Two participants worried about the feasibility of such a robot vacuum cleaner. Another said that if it could be trusted with respect to cleaning performance, it would be a great solution as it would really take over the cleaning job. Signs of anthropomorphism and personality were observed throughout the evaluations. One participant explicitly mentioned that he experienced the robot vacuum cleaner as having a distinctive character. Out of fifteen participants, fourteen assigned a gender to the robot vacuum cleaner. When talking about it, they frequently referred to it as 'he' or 'him'. Only one participant consequently used 'the robot vacuum cleaner' or 'it'. Participants said that it felt as if the robot vacuum cleaner was alive (3 participants), and like a domestic animal or a $\operatorname{dog}(3)$, or even like an infant (1).

The results with respect to the task of rating the personality characteristics indicate that the perceived personality matches with the intended product personality. Of the fifteen participants, twelve perceived the robot vacuum cleaner as calm. They agreed with perceiving this characteristic (10 participants), or even strongly agreed (2). Cooperative yielded similar results (10 participants agreed, 2 strongly agreed). Again, twelve participants thought that the vacuum cleaner behaved in a systematic way (4 participants agreed, whereas 8 strongly agreed). And another twelve thought that it liked routines (4 participants agreed, whereas 8 strongly agreed). Then, the robot vacuum cleaner was generally perceived as being polite ( 7 participants agreed, 2 others strongly agreed). The participants were less outspoken 
Fig. 4 The results of six of the characteristics
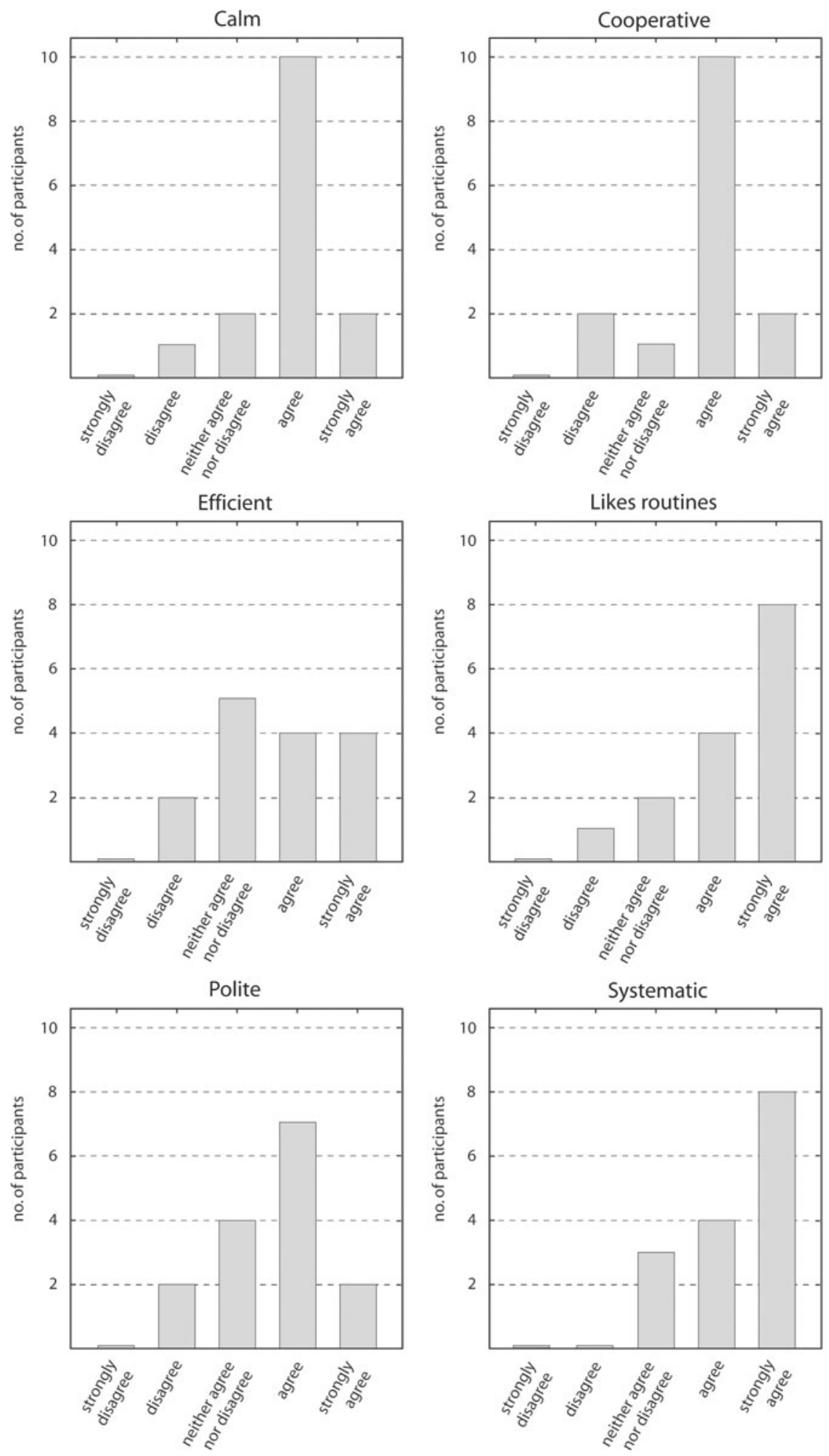

with respect to how efficient they perceived the robot vacuum cleaner. Figure 4 provides an overview of the results for these six personality characteristics.

Some of the responses by the participants were due to prototype effects. These are deviations from the original idea as intended by the designer. These effects deal with the fact that the video prototype is limited in time, and limited in situations. Not everything a robot vacuum cleaner might encounter could be implemented. But some of the remarks went beyond the limited set of situations. What if there is 
nobody to help to empty the robot? What if it sucked up jewelry? And those situations that were selected for implementation happen in rapid succession; a complete vacuum cleaning cycle in only five minutes is rather quick for a real robot vacuum cleaner. This led some of participants to comment upon the robot vacuum cleaner's 'amazing' recharging capabilities for example.

\section{Conclusion and Discussion}

People anthropomorphize robot vacuum cleaners and, as an aspect of this, they attribute personality characteristics. Designers can make use of this phenomenon by deliberately designing a robot vacuum cleaner personality. This, by going through a process of determining personality, developing behavior in an iterative way and evaluating the user experience of the robot vacuum cleaner. We recommend using a personality model as a tool for developing robot behavior. We found it useful as a guideline when taking design decisions and helpful in developing consistent behavior.

We have investigated what kind of personality is desired for a robotic vacuum cleaner and found that people prefer a calm, polite, and cooperative robot vacuum cleaner that works efficiently, systematically and likes routines. Based on these findings, we developed its behavior and evaluated it with end users by means of a video prototype. The results of our evaluation indicate that people recognized the intended personality in the robot's behavior.

We think that the designed personality is beneficial with respect to the user experience, as it helps people to form a conceptual model of the robot vacuum cleaner. It leads to a consistent set of behaviors, across situations over time. When users learn to know the personality of their robot, they are able to interact in an appropriate way and to predict how the robot responds. Furthermore, it is important to determine a desired robot vacuum cleaner personality, and implement-from a user point of view—desired behavior. For the user experience it is important to focus on the subjective aspects of human-robot interaction (such as the perception of calm, cooperative behavior), instead of considering objective measures such as vacuuming speed and efficiency only. This does not mean that cleaning performance is unimportant. As we have seen, people expect a robot vacuum cleaner to fulfill a user need: having a clean floor.

In future research, we want to investigate the effect of a deliberately designed robot personality on the user experience in more detail. Do people have a good understanding of the robot? Do people have trust in the robot? Also, we want to compare multiple personalities and the effect thereof on the user experience of robot vacuum cleaner behavior. And, as watching a video prototype of robot vacuum cleaner behavior is not the same as living with such a robot, we suggest doing a longitudinal study on the user experience with a real robot vacuum cleaner, in a real domestic setting.

Open Access This article is distributed under the terms of the Creative Commons Attribution Noncommercial License which permits any noncommercial use, distribution, and reproduction in any medium, provided the original author(s) and source are credited.

\section{References}

1. Meerbeek B, Saerbeck M, Bartneck C (2009) Iterative design process for robots with personality. In: Proceedings of the new frontiers in human-robot interaction, symposium at the AISB2009 convention, SSAISB. Springer, Berlin, pp 94-101. ISBN-190295680X

2. Rutland J (1978) Exploring the world of robots. Pan Books, London, pp 20-21

3. Prassler E, Kosuge K (2008) Domestic robots. In: Siciliano B, Khatib O (eds) Springer handbook of robotics. Springer, Berlin, pp 1253-1281

4. Sung J, Grinter RE, Christensen HI (2010) Domestic robot ecology: an initial framework to unpack long-term acceptance of robots at home. Int J Soc Robot 2:417-429

5. Sung J, Grinter RE, Christensen HI, Guo L (2008) Housewives or technophiles?: understanding domestic robot owners. In: Proceedings of the 3rd ACM/IEEE international conference on humanrobot interaction 2008, Amsterdam, The Netherlands, pp 129-136

6. Sung J, Guo L, Grinter RE, Christensen HI (2007) "My Roomba is Rambo": intimate home appliances. J Ubi Comput 4717:145-162

7. Young JE, Hawkins R, Sharlin E, Igarashi T (2009) Toward acceptable domestic robots: applying insights from social psychology. Int J Soc Robot 1:95-108

8. Forlizzi J (2007) How robotic products become social products: an ethnographic study of cleaning in the home. In: Proceedings of the 2nd ACM/IEEE international conference on human-robot interaction 2007, pp 129-136

9. Forlizzi J, DiSalvo C (2006) Service robots in the domestic environment: a study of the Roomba vacuum in the home. In: Proceedings of the ACM/IEEE conference on human-robot interaction 2006, Salt Lake City, Utah, USA, pp 265-285

10. McCrae RR, Costa PT (1987) Validation of the five-factor model across instruments and observers. J Pers Soc Psychol 52:81-90

11. Costa PT, McCrae RR (1992) NEO PI-R professional manual. Psychological Assessment Resources, Odessa

12. Gosling SD, Rentfrow PJ, Swann WB (2003) A very brief measure of the Big Five personality domains. J Res Pers 37:504-528

13. Govers PCM (2004) Product personality. PhD, Delft University of Technology

14. Órtiz-Nicolás JC (2006) Product personality in interaction. MSc, Delft University of Technology

15. Reeves B, Nass C (1996) The media equation. Cambridge University Press, Cambridge

16. Meerbeek BW, Hoonhout HCM, Bingley P, Terken J (2008) The influence of robot personality on perceived and preferred level of user control. Interact Stud 9(2):204-229

17. Norman DA (2004) Emotional design: why we hate (or love) everyday things. Basic Books, New York

18. Van Loenen E, De Ruyter B, Teeven V (2006) ExperienceLab: facilities. In: Aarts E, Diederiks E (eds) Ambient lifestyle: from concept to experience. BIS, Amsterdam, pp 47-53

19. Dautenhahn K (2007) Methodology and themes of human-robot interaction: a growing research field. Int $\mathrm{J}$ Adv Robot Syst 4(1):103-108 
20. Walters ML, Syrdal DS, Dautenhahn K, TeBoekhorst R, Koay KL (2008) Avoiding the uncanny valley: robot appearance, personality and consistency of behavior in an attention-seeking home scenario for a robot companion. Auton Robots 24(2):159-178

21. Boess SU (2008) First steps in role playing. In: Proceedings of the ACM conference on human factors in computing systems, Florence, Italy, pp 2017-2024

22. Klooster S, Overbeeke CJ (2005) Designing products as an integral part of choreography of interaction: the product's form as an integral part of movement. In: Proceedings of the conference on design and semantics of form and movement 2005, pp 23-35

23. Van Egmond R (2005) Emotional experience of frequency modulated sounds: implications for the design of alarm sounds. In: de Waard D, Brookhuis KA, Weikert CM (eds) Human factors in design. Shaker, Maastricht, pp 1-12

Bram Hendriks obtained his MSc Design for Interaction degree at the Faculty of Industrial Design Engineering of the Delft University of Technology. He also studied Industrial Design at the Eindhoven University of Technology and the Politecnico di Milano. For his graduation project at Philips Research, he studied the user experience of a robot vacuum cleaner. He has a strong user focus and is interested in combining insights from design, engineering and the social sciences into new solutions.
Bernt Meerbeek holds a MSc in Information Management from Tilburg University and a PDEng in User-System Interaction from Eindhoven University of Technology. Since 2005, he works at Philips Research on user-system interaction in various projects-including iCat and robot vacuum cleaners-and is highly involved in User Experience research at the ExperienceLab facility.

Stella Boess is assistant professor at the Delft University of Technology. She teaches user research in and for design. She graduated in product design at the HDK Saar and received her PhD degree at Staffordshire University.

Steffen Pauws holds a MSc in Medical Informatics from Leiden University and a PDEng in Software Technology and a PhD, both from Technical University of Eindhoven, the Netherlands. Since 2000, he has been with Philips Research as a senior scientist covering various research areas including algorithms and user interfaces for music retrieval.

Marieke Sonneveld is assistant professor at the Delft University of Technology and has her own studio: Tactile Affairs. She graduated and received her $\mathrm{PhD}$ at the Delft University of Technology. Her research domain is the tactual experience in human product interaction, especially the affective aspects of this experience in the context of health and well-being. 